\title{
Color Theme-based Aesthetic Enhancement Algorithm to Emulate the Human Perception of Beauty in Photos
}

\author{
KAREN PANETTA and LONG BAO, Tufts University \\ SOS AGAIAN, The City University of New York \\ VICTOR OLUDARE, Tufts University
}

\begin{abstract}
Fine Art Photography is one of the most popular art forms, which creates lasting impressions that elicit various human emotional reactions. Photo aesthetic enhancement aims at improving the aesthetic level of the photo to please humans by updating color appearance or modifying the geometry structure of objects within that photo. Even though several aesthetic enhancement methods have been proposed, to our knowledge, there is no research to explore, highlight, and accentuate photos' intrinsic aesthetic value to elicit a stronger response from the human observer about the photos' theme. To meet this challenge, a new multimedia technology called automatic color theme-based aesthetic enhancement (CT-AEA) is proposed by leveraging big online data to perform timely collection and learning of humans' current aesthetic perception-behavior over photos and color themes in art, fashion, and design. Unlike existing aesthetic enhancement that examines the composition, such as the geometric structure of the image contents and color/luminance-related (color tone and luminance distribution) characteristics, this CT-AEA takes into consideration the importance of a suitable color theme, namely a set of dominant colors for the design when assessing the aesthetic appearance of a photo. This algorithm is composed of (1) utilizing the knowledge gained from the human evaluator's perception of beauty from existing online datasets, rather than simply applying prior existing knowledge of color harmony theory; (2) developing a new color theme difference equation that exhibits order-invariance and percentage-sensitive properties; (3) designing an optimal color theme recommendation to maximize the aesthetic performance, while minimizing the color modification cost to solve the problems of color inconsistencies and distortion. Experimental results, quantitative measure, and comparison tests demonstrate the algorithm's effectiveness, advantages, and potential for use in many color-related art and design applications.
\end{abstract}

\section{CCS Concepts: • Applied computing $\rightarrow$ Media arts;}

Additional Key Words and Phrases: Aesthetic enhancement, color theme, fine art photography, human aesthetic perception, big data

\section{ACM Reference format:}

Karen Panetta, Long Bao, Sos Agaian, and Victor Oludare. 2019. Color Theme-based Aesthetic Enhancement Algorithm to Emulate the Human Perception of Beauty in Photos. ACM Trans. Multimedia Comput. Commun. Appl. 15, 2s, Article 62 (July 2019), 17 pages.

https://doi.org/10.1145/3328991

Authors' addresses: K. Panetta and L. Bao (corresponding author) are with Department of Electrical and Computer Engineering, Tufts University, Medford, MA 02155 USA; emails: karen@ece.tufts.edu, baolonghnu@gmail.com; S. Agaian is with Department of Electrical Engineering, University of Texas at San Antonio, San Antonio, TX 78249 USA; email: Sos.Agaian@csi.cuny.edu.

Permission to make digital or hard copies of all or part of this work for personal or classroom use is granted without fee provided that copies are not made or distributed for profit or commercial advantage and that copies bear this notice and the full citation on the first page. Copyrights for components of this work owned by others than ACM must be honored. Abstracting with credit is permitted. To copy otherwise, or republish, to post on servers or to redistribute to lists, requires prior specific permission and/or a fee. Request permissions from permissions@acm.org.

(C) 2019 Association for Computing Machinery.

1551-6857/2019/07-ART62 \$15.00

https://doi.org/10.1145/3328991 


\section{INTRODUCTION}

Photography has not existed as long as other art forms, such as painting, music, and crafts, but it still appears pleasing or attractive to the eye and can inspire a person to convey a story or create an atmosphere $[1,2]$. A person may feel a chill through their body after viewing a winter snow scene, an invigorating "fresh" feeling from viewing a photo of a green tree or be eager to buy a product from viewing a commercial photo. However, during its short history, the development of camera technologies and image processing has only significantly improved the quality and ease of capturing photographs, even within mobile devices. Hence, it is still a challenge to solve the understanding of the perception of beauty in photography, since even quality images may fail to elicit an emotional response from the observer, in terms of providing aesthetic enjoyment [3, 4]. It is still unclear how humans, as individuals, extract different interpretations from photos and have various emotional reactions to an image [5]. To meet this challenge, many researchers have been consistently working to make computers understand and design "art" [6-8].

According to the theory of color psychology, the aesthetic level of a photo is related to the degree of "pleasing to the eyes." For a picture, most individuals may only care about how soothing it is, whereas photographers may need to consider its composition and color/luminance distribution [9, 10]. Based on these two key factors, existing aesthetic enhancement algorithms are divided into two groups: the composition-related approach $[11,12]$ and the color/luminance related approach.

The composition-related approach focuses on the relationship of objects within the image and the geometric structure of the objects. One way to accomplish this is to crop the images $[4,13,14]$. By cutting additional edge contents, the most visually important region in an image is retained with good geometric structure. Another approach is to relocate objects within images [15-17]. As shown in Figure 1(d), the basic idea of this approach is to extract the foreground objects and then relocate them to make a good geometric structure meeting the aesthetic requirement. To implement this concept, work has been proposed to use an optimization framework to achieve cropping and retargeting operations [18]. Following this original research work, two research papers were published on using a learning-based algorithm to improve the performance of retargeting $[8,19$, 20]. The state-of-the-art research paper proposed by Zhang utilized dependence analysis-based object decomposition to adjust objects' positions while keeping the original scene content [21]. As a conclusion of the composition-related approach, there were two remaining challenges: (1) inconsistency in the background when the foreground objects are moved; (2) interrelations among the complex foreground objects.

Another aesthetic enhancement approach relates to colors/luminance adjustment. To achieve the color/luminance-related aesthetic enhancement, one traditional way is to adjust the tone of photos [22]. By utilizing the learning experience of a high-quality reference dataset, supervised machine learning is applied to achieve an automatic global adjustment [21, 23, 24]. However, its performance is limited to changing only the contrast, brightness, and saturation, as shown in Figure 1(c). Another approach is to utilize color transfer methods. Color transfer is an efficient tool to transfer source colors into a target photo/image [25-27], making the target image present a similar color as the source colors. By manually selecting source colors, an original photograph will be recolored to generate a recolored imagethat shares the same content information with the original photograph and the same colors as the selected source colors [28]. Using different source color combinations, as a color theme, generated a different color appearance. However, a proper selection of source colors required a professional designer with the experienced skills and knowledge about the color. Otherwise, the recolored image will not present a "pleasing and harmonious" or aesthetic appearance.

However, both these existing composition-related and color/luminance-related approaches are not intended to explore, highlight, and accentuate photos' intrinsic aesthetic value to elicit a 


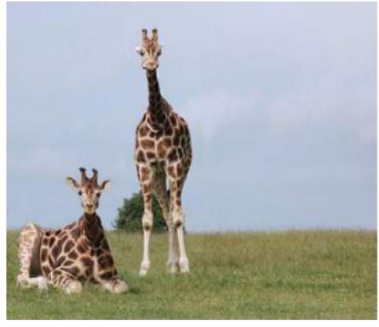

(a)

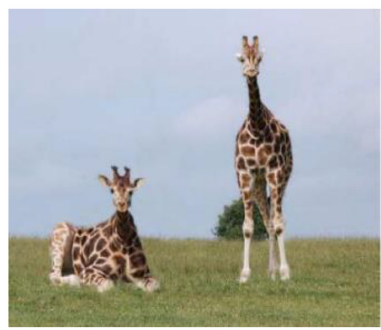

(d)

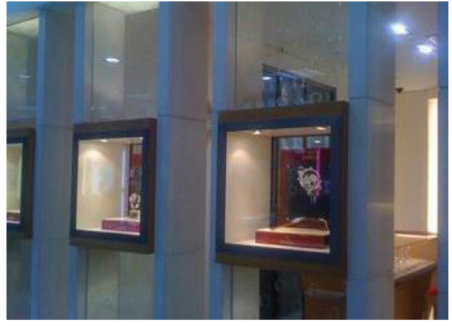

(b)

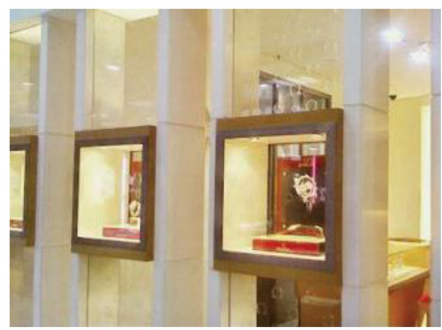

(e)

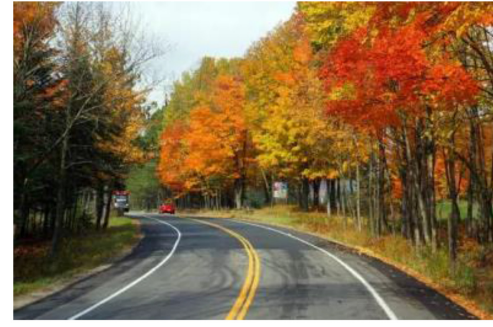

(c)

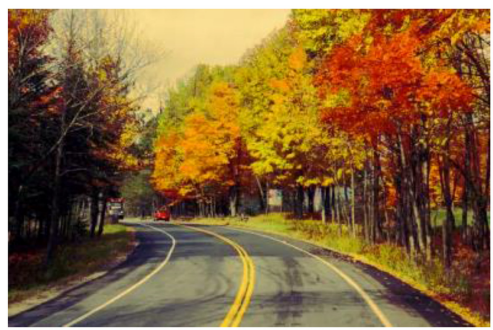

(f)

Fig. 1. Examples of aesthetic enhancement methods. The top row are the original images and the bottom row presents the aesthetically enhanced images: (a)-(c) original images; (d) enhanced image by the compositionbased method [20]; (c) enhanced image by the tone-based method [23]; (f) enhanced image by the proposed CT-AEA to visually demonstrate that the CT-AEA method enhances the prominence of the colors to elicit the ambiance of a vibrant autumn day.

stronger response from the human observer about the photos' theme, as shown in Figure 1(f). Also, no research has recognized the key role the color theme plays in determining a photo's aesthetic level and its impact on eliciting an emotional response from the human observer [29, 30]. Most importantly, existing photo aesthetic enhancement approaches are all based on existing color psychology knowledge and experience, which are collected on a small survey scale. Furthermore, humans' aesthetic perception and personal tastes in photography changes over time, and existing methods do not take this into consideration to update their algorithm.

To accomplish this task, big online data is leveraged to survey current human aesthetic perception on a large scale. From this, a new color theme-based aesthetic enhancement algorithm (CT-AEA) is proposed that is different from the tone-based and composition-based methods. By finding the inherent color theme of an input photo, humans' aesthetic experience for different color themes on a large online dataset is applied to generate an optimal color theme. This color theme will be utilized to update the color distribution of the input photo by enjoying a color theme update equation. Experimental results show that the proposed CT-AEA method performs well at achieving the maximum aesthetic enhancement with a minimized cost of color modification within images and their color themes.

\section{COLOR THEME-BASED APPROACH}

Early stages of visual psychology revealed that different colors will create different emotional responses and feelings in a human's visual perception [31,32]. The color green is typically associated with freshness, nature, life, and energy; while the color red will present the feelings of love, passion, heat, rage, and anger. Further study shows that color combinations used as a color theme is important in presenting aesthetic enjoyment and eliciting different feelings in humans [33, 34]. 


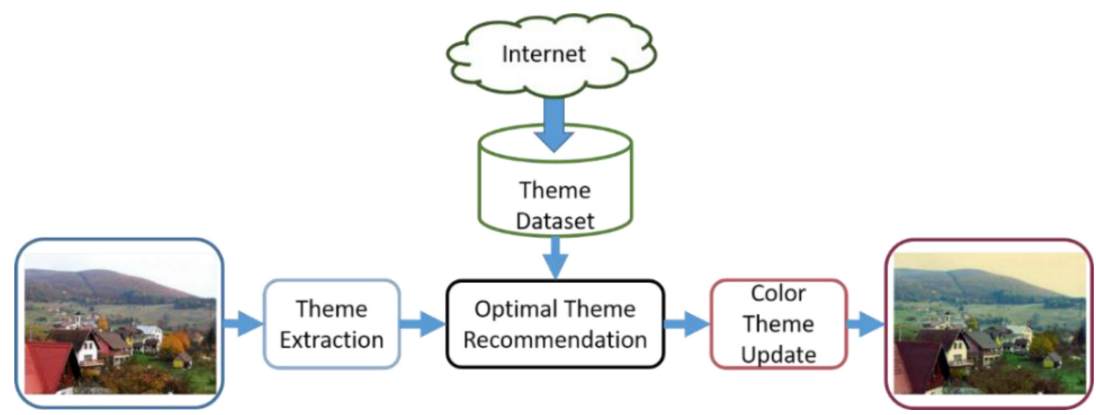

Fig. 2. Framework of the proposed color theme-based aesthetic enhancement algorithm (CT-AEA): including (1) a theme extraction process to find the inherent color theme $(T)$ of an input photograph by utilizing clustering technology as shown in Equation (1); (2) a theme dataset used to collect human score $(\mathcal{H}(\widehat{\mathrm{T}}))$ about the human experience concerning different color themes $(\widehat{\mathrm{T}}) ;(3)$ an optimal theme recommendation, which is used to find the most similar color theme $\left(T^{*}\right)$ with the highest human score by Equation (4), based on the extracted inherent color theme and a new color theme difference equation; (4) a color theme update process used to update the colors of the input photo based on the recommended optimal color theme by Equation (10).

Some color themes will create a pleasing and harmonious emotional response, while some color themes will not. Hence, according to these psychological observations, the color theme is key to revealing the secret of how humans feel as a result of viewing a photograph. This is a reason why designers treat color themes so importantly throughout the entire art creation and design processes. A photograph will present a different aesthetic feeling or strengthen its original one by changing or modifying the photograph's color theme. This is a fundamental assumption of the proposed color theme-based approach.

To implement this color theme-based approach, one necessary step is to determine and assess how a color theme manipulates the human observer's aesthetic feelings. By practicing over decades, scientists proposed different color theme templates as guides in selecting suitable color combinations. These templates include the Kular template and the Matsuda template. The Kular template focuses on specifying a fixed angular distance on a color wheel between each color in one color theme, whereas the Matsuda template replaces the fixed angle distance with sectors to include more variations. These two templates have been utilized for decades, especially for graphics. However, based on a recent discovery gained from exploring large datasets containing human scores for thousands of color themes on the websites of Kular and Colourlover, these two templates are not always correct [35]. Hence, in this article, the knowledge of the Kular and Matsuda templates are not applicable. Instead, utilizing machine learning, the experience from these online datasets is learned and applied in the proposed algorithm.

\section{AESTHETIC ENHANCEMENT ALGORITHM}

As shown in Figure 2, the proposed color theme-based aesthetic enhancement algorithm (CT-AEA) is composed of four blocks, including theme extraction, optimal theme recommendation, theme dataset, and color theme update.

\subsection{Theme Extraction}

Since the goal of this proposed CT-AEA aims at accentuating the input photograph's intrinsic ambiance, a first step should be to determine and measure what properties elicit a human's emotional feeling in response to viewing an image. However, quantitatively capturing an abstract concept 
such as a human feeling is not currently feasible. An alternative approach is to locate a color theme that is known to be affiliated with eliciting different human feelings. This color theme extraction can be described as a clustering problem to divide all the color pixels within the input photo into their natural color groups. Then, the central color of each color group is combined to form a color theme.

To perform this clustering task, a Fuzzy C-means (FCM) method is utilized [36, 37]. The FCM method was proposed by J. C. Dun in 1973 and improved by J. C. Bezdek in 1981. The special advantage of FCM is to allow one piece of data to belong to two or more clusters. Hence, for each data point, this algorithm will provide its membership information to each cluster center [36, 37]. This soft clustering will be beneficial in reducing the color artifacts in the following color transfer, as shown in Equation (10). The goal of FCM is to minimize an objective function, as shown in Equation (1).

$$
\underset{T}{\arg \min } \sum_{k=1}^{K} \sum_{i=1}^{w} \sum_{j=1}^{l} \omega_{i, j, k}^{m}\left\|I_{i, j}-T_{k}\right\|^{2},
$$

where $I_{i, j}$ is a RGB color vector of pixels in input image/photo with a size of $w \times$ $l, I_{i, j}=\left[I_{i, j}^{R}, I_{i, j}^{G}, I_{i, j}^{B}\right] ; T_{k}$ is the center of $k$ th group, which is calculated by Equation (2), $T=$ $\left[T_{1}, T_{2}, \ldots T_{k}, \ldots T_{K}\right], T_{k}=\left[T_{k}^{R}, T_{k}^{G}, T_{k}^{B}\right] ; \omega_{i, j, k}^{m}$ is the degree of membership of $I_{i, j}$ belonging to the group $T_{k}$, which can be calculated in Equation (3). $k$ is the number of clustered groups.

$$
\begin{gathered}
T_{k}=\sum_{i=1}^{w} \sum_{j=1}^{l} \omega_{i, j, k}^{m} I_{i, j} / \sum_{i-1}^{w} \sum_{j=1}^{l} \omega_{i, j, k}^{m}, \\
\omega_{i, j, k}^{m}=1 / \sum_{d=1}^{K}\left(\frac{\left\|I_{i, j}-T_{k}\right\|}{\left\|I_{i, j}-T_{d}\right\|}\right)^{2 /(m-1)},
\end{gathered}
$$

where $m$ is the fuzzifier. A larger value of $m$ means a larger overlap between the groups. The default value is empirically set as 2 .

In each iterative operation of this FCM, the cluster centers are calculated by Equation (2), then the weights are updated by Equation (3), and finally, the value of an objective function is calculated as shown in Equation (1). By this iterative process, the value of this objective function will be minimized. Once the improvement of this iterative operation about the objective function is smaller than a threshold value, this FCM terminates. A threshold value of $10^{-5}$ was chosen experimentally.

This FCM will output two important matrices: a weight matrix and a center matrix. The center matrix carries data of the intrinsic color theme of the input photo/image. The weight matrix will be utilized in a color theme update equation, as shown in Equation (10).

\subsection{Theme dataset}

As shown in Figure 1, the theme dataset is a connection between online Internet data and the CT-AEA. The function of this theme dataset block is to collect different color theme data and their corresponding human scores from online websites, including the Kular and Colourlover websites. These websites post different color themes containing five different colors and collect human scores by counting its "heart," where one "heart" means one user likes the color theme design. In our design, this theme dataset is connected in real time to these important websites to timely collect information of humans' responses to these color themes, considering the change of humans' aesthetic trend over time. However, in this research article, to present results compared to a known benchmark, the downloaded Kular and Colourlover image datasets from the website of the Dynamic Graphics Project lab of the University of Toronto are used as the theme datasets [35]. The 
output of the theme dataset is a collection of a five-color theme $(\hat{\mathrm{T}})$ and its average human score $\mathcal{H}(\hat{\mathrm{T}}))$. Since the online websites only contain themes of five colors, the original inherent theme will also be five colors, meaning that the number of groups $(K)$ in the FCM equals to 5 .

\subsection{Optimal Theme Recommendation}

The optimal theme recommendation process finds a color theme within the theme dataset that meets two requirements: (1) it should have a high human score, ensuring a high aesthetic level; and (2) it should be close to the original inherent theme, meaning that it highlights the original human feeling rather than changing it to a totally different ambiance. Hence, the goal of optimal theme recommendation is to minimize a cost function in Equation (4), containing two evaluation components: theme difference calculation $\mathcal{D}\left(T^{*}, T\right)$ and human score $\mathcal{H}\left(T^{*}\right)$.

$$
\exists T^{*} \in \widehat{\mathrm{T}} \underset{T^{*}}{\arg \min } \mathcal{D}\left(T^{*}, T\right)-\mathcal{H}\left(T^{*}\right),
$$

where $T^{*}$ represents the optimal theme matrix, containing five dominant colors, where each color is represented by three R, G, B component values, as shown in Equation (5), in a theme dataset $\widehat{\mathrm{T}} ; T$ represents the inherent color theme generated by Equation (6) with central information from Equation (2).

$$
\begin{aligned}
T^{*} & =\left[\begin{array}{lllll}
R_{1}^{*} & R_{2}^{*} & R_{3}^{*} & R_{4}^{*} & R_{5}^{*} \\
G_{1}^{*} & G_{2}^{*} & G_{3}^{*} & G_{4}^{*} & G_{5}^{*} \\
B_{1}^{*} & B_{2}^{*} & B_{3}^{*} & B_{4}^{*} & B_{5}^{*}
\end{array}\right] \\
T & =\left[\begin{array}{lllll}
C_{1}^{R} & C_{2}^{R} & C_{3}^{R} & C_{4}^{R} & C_{5}^{R} \\
C_{1}^{G} & C_{2}^{G} & C_{3}^{G} & C_{4}^{G} & C_{5}^{G} \\
C_{1}^{B} & C_{2}^{B} & C_{3}^{B} & C_{4}^{B} & C_{5}^{B}
\end{array}\right]
\end{aligned}
$$

In the theme dataset, the human score $\mathcal{H}\left(T^{*}\right)$ is a fixed value within a range of [0,5], where a higher value means the better the aesthetic level. Hence, in the cost function, this human score $\mathcal{H}\left(T^{*}\right)$ is multiplied by -1 in the calculation to make a minimized cost function represent an optimal theme. For the theme difference calculation in Equation (4), it should possess two properties: order-invariance and percentage-sensitive. The order-invariant property means that the order of five colors in a color theme should generate the same difference value between the two themes. To achieve this property, the inherent color theme will be compared to each permutation by colors within a color theme in the theme dataset. The minimum difference of all possible permutations will be set as a final difference between these two color themes. To calculate the difference between two color themes, the L1 norm is adapted [38].

The percentage-sensitive property is to ensure that an optimal color theme will not change the color appearance of the original input photograph significantly, resulting in presenting another ambiance rather than its original one. Hence, a color within the inherent color theme, which takes a larger percentage of color pixels in the input photographs, should be assigned a larger weight in the theme difference calculation.

Considering these two properties, the difference equation $\mathcal{D}\left(T^{*}, T\right)$ in Equation (4) is calculated by Equation (7).

$$
\mathcal{D}\left(T^{*}, T\right)=\min \left(\left\{\left|\left(P^{c}\left(T^{*}\right)-T\right) \times p_{1}\right| \mid P^{c}\left(T^{*}\right) \in \widehat{\mathrm{P}^{c}}\left(T^{*}\right)\right\},\right.
$$

where $P^{c}\left(T^{*}\right)$ is one permutation case among all possible permutation of color theme $\left(\widehat{\mathrm{Pc}^{c}}\left(T^{*}\right)\right)^{\text {“ }} \mathrm{X}^{\text {” }}$ represents a matrix multiplication; $p$ is a column percentage vector as defined in Equation (8) with 
the size of $K * 1$.

$$
\begin{aligned}
& p(k, 1)=\sum_{i=1}^{w} \sum_{j=1}^{l} N_{i, j}^{k} / w * l, \quad k \in[1, K], \\
& N_{i, j}^{k}= \begin{cases}1 & \omega_{i, j, k}^{m}=\max \left(\omega_{i, j, 1: K}^{m}\right), \\
0 & \text { others }\end{cases}
\end{aligned}
$$

where function $\max \left(\omega_{i, j, 1: K}^{m}\right)$ is to calculate the maximum degree of membership belonging to each groups in Equations (1)-(3).

\subsection{Color Theme Update}

The purpose of the color theme update block in Figure 2 is to transfer the located optimal color theme into the input photograph. Successful color theme update needs to ensure there are no color artifacts generated in the outputted results. The soft FCM clustering is key to achieving this by providing a degree matrix of membership belonging to each group. By utilizing this information, the color theme update will smoothly update the colors in Equation (10).

$$
\left[\begin{array}{c}
E_{i, j}^{R} \\
E_{i, j}^{G} \\
E_{i, j}^{B}
\end{array}\right]=\left[\begin{array}{c}
I_{i, j}^{R} \\
I_{i, j}^{G} \\
I_{i, j}^{B}
\end{array}\right]+\left(T^{*}-T\right) \times\left[\begin{array}{c}
\omega_{i, j, 1}^{m} \\
\omega_{i, j, 2}^{m} \\
\omega_{i, j, 3}^{m} \\
\omega_{i, j, 4}^{m} \\
\omega_{i, j, 5}^{m}
\end{array}\right],
$$

where $\left[E_{i, j}^{R}, E_{i, j}^{G}, E_{i, j}^{B}\right]$ are the R, G, B component values of a color pixel in the position $(i, j)$ of the output enhanced photo.

\section{EXPERIMENTAL RESULTS}

CT-AEA transfers a real image captured by a commercial camera into an aesthetic image, which can be used as a postcard, a cover photo of a magazine, or an art piece. This section will present several experimental results and quantitative measure to demonstrate the effectiveness of the CTAEA in accentuating the aesthetic feeling of photographs.

\subsection{Art Photography}

Figure 3 shows the experimental results of updating the color of art images. As shown in Figure 3(b), compared to the original photo in Figure 3(a), the processed photograph carries a much more artistic color distribution presenting a much more interesting photo for display. Another example is shown in Figures 3(c) and 3(d). The original image in Figure 3(c) is clear, whereas the transferred image presents a more exciting feeling of fresh, peaceful, and spring in Figure 3(d). As another example, a photo of a painting in Figure 3(e) is tested and processed to be as shown in Figure 3(f). As shown in Figure 3(e), the original image depicts a rainy city. However, the feeling of "raining" is not so strong to elicit a human's emotional response. To solve this, the CT-AEA is applied, and the transferred photo presents a much stronger chilly feeling of rain, as shown in Figure 3(f). Also, this chilly feeling is stronger in the recolored result in Figure 3(f) than its original image in Figure 3(e), making the recolored results portray some artistic enjoyment for humans. The similar artistic enhancement can be also observed from another original photo in Figure 3(g) and its recolored result in Figure 3(h). These examples demonstrate the effectiveness of the proposed CT-AEA in exploring and strengthening the inherent aesthetic feeling of the input image. 


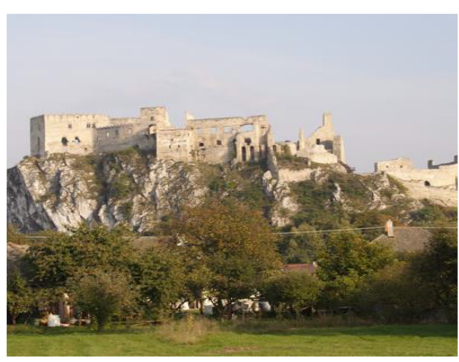

(a)

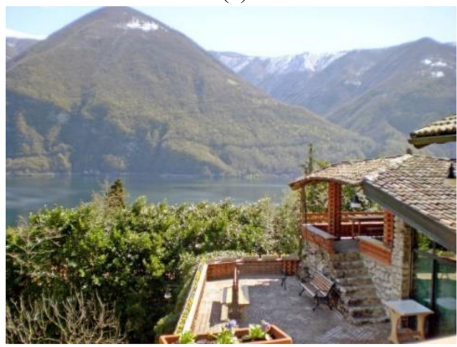

(c)

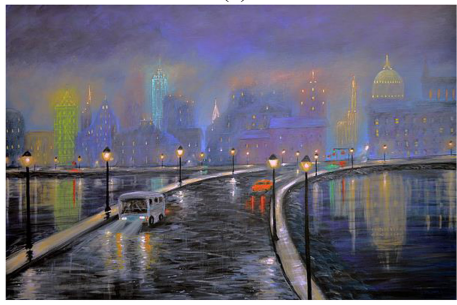

(e)

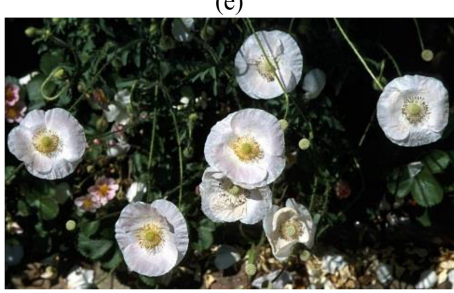

(g)

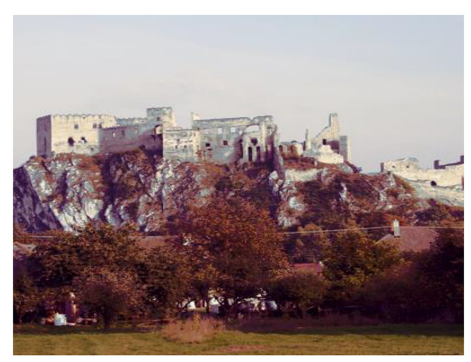

(b)

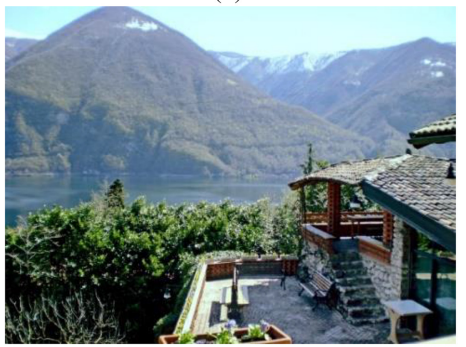

(d)

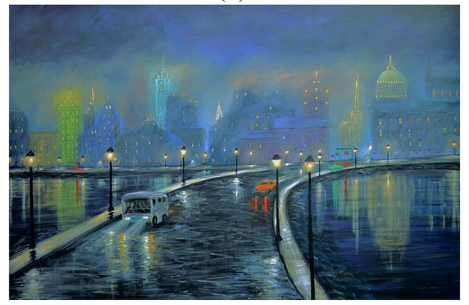

(f)

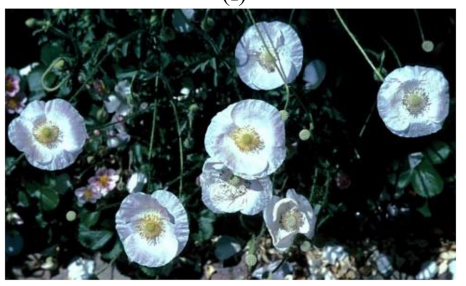

(h)

Fig. 3. Experimental results of original photo image: (a), (c), (e), and (g) are the original images; (b), (d), (f), and $(h)$ are the artists' images transferred by the proposed CT-AEA. Image (b) is much more artistic than Image (a) ${ }^{1}$ to be selected as a photo for display; Image (d) shows a stronger feeling of fresh, peaceful, and spring than its original image in (c) $)^{2}$; Image (f) provides a stronger feeling of rainy than (e) ${ }^{3}$; Image (h) provides a more artistic atmosphere. Here, we show visual samples of the results, and their corresponding quantitative measures are provided in Section 4.4).

\subsection{Travel Photography}

The travel industry has played an essential role in commerce. This proposed aesthetic enhancement can support travel industry marketing by showing the beauty of natural scenes to travel

\footnotetext{
${ }^{1}$ The original image is downloaded from https://theodora.com/wfb/photos/slovakia/slovakia_photos_9.html.

${ }^{2}$ The original image is downloaded from https://www.booking.com/hotel/it/il-rustico.sv.html.

${ }^{3}$ Misty City is a photon of a painting by Ken Figurski that was uploaded to the website https://fineartamerica.com/featured/ misty-city-ken-figurski.html on December 5, 2015.
}

ACM Trans. Multimedia Comput. Commun. Appl., Vol. 15, No. 2s, Article 62. Publication date: July 2019. 


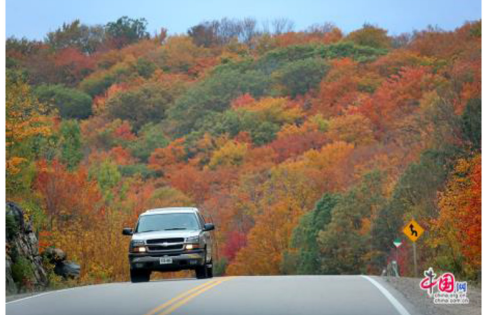

(a)

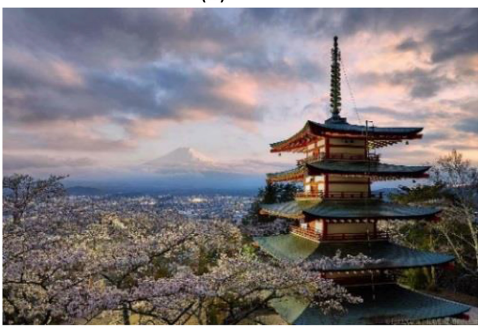

(c)

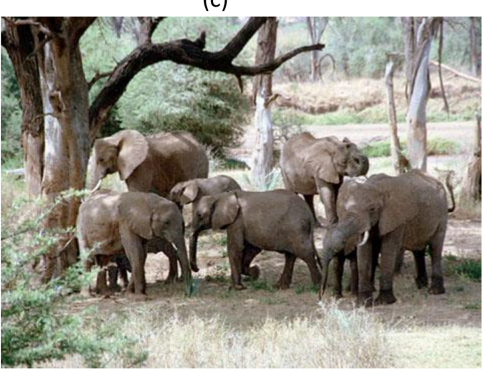

(e)

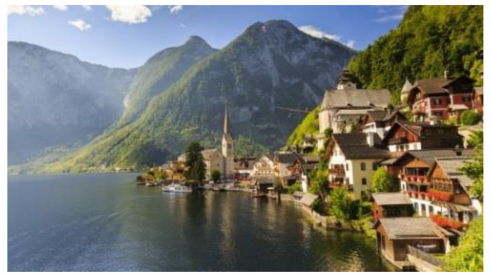

(g)

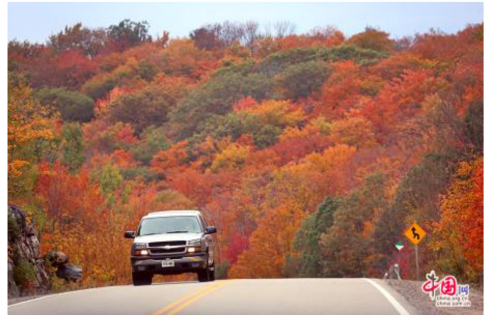

(b)

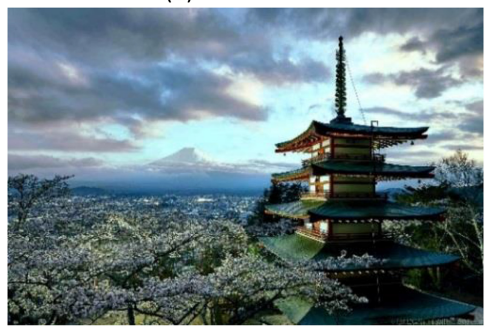

(d)

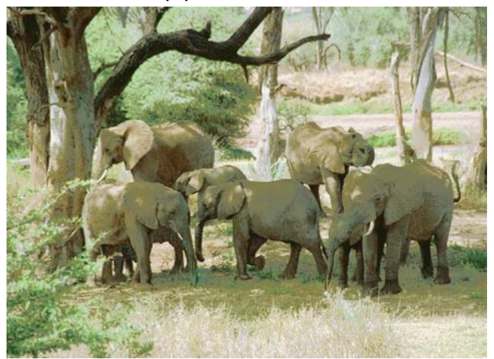

(f)

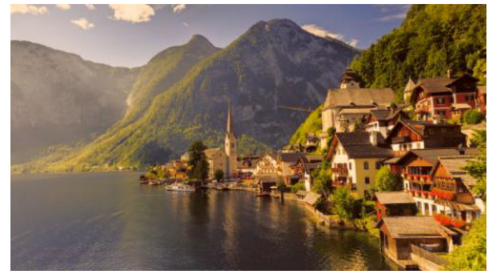

(h)

Fig. 4. Simulation result of travel photography: (b) recolored image by CT-AEA makes a more appealing appearance than original image $(\mathrm{a})^{4}$; (d) recolored image shows more color harmony than original image (c) [39]; (f) recolored image shows a more beautiful image than (e) [39] by presenting the feeling of fresh green; (h) recolored image shows a strong feeling of warmth as the sun sets, while the clear original image (g) [39] presents a neutral feeling, demonstrating the performance of CT-AEA in enriching the colorfulness.

destinations to attract tourists or even share the beauty of what tourists see during their travels via social media. As an example in Figure 4(a), the original image is clear, but not so colorful. By the CT-AES, the colors are enriched and the scene becomes alive and attractive. As another example, CT-AES can transfer the original image in Figure 4(c) to an image with appropriate color harmony in Figure 4(d) to elicit the feeling of peace. Also, CT-AES can make the recolored image

\footnotetext{
${ }^{4}$ The original image is downloaded from http://guoqing.china.com.cn/zhuanti/node_7221892.htm.
} 
in Figure 4(f) show a more beautiful image than its original image in Figure 4(e) by presenting the feeling of life from fresh green, and make the recolored image in Figure 4(h) show a strong feeling of the warmth of a sunset, while the clear original image in Figure 4(g) presents a neutral feeling. Hence, this CT-AEA shows a good performance in enriching colorfulness to make it beautiful and appealing.

\subsection{Food Photography}

Tempting food advertisements should have an impactful visual appearance to whet the viewer's appetite. One of the key features attracting an individual's appetite for food is the colorfulness of the food within images [40]. A color-enriched food photo will be much more impactful at attracting people's attention and willingness to purchase the food. CT-AEA can support this color-enriching task as shown in Figure 5. Compared to the original image, the recolored image is very colorful and appealing, especially presenting the fresh green of vegetables in Figures 5(d), 5(f), and 5(h), the fresh yellow of an orange in Figure 5(b), and the fresh red of beef in Figure 5(d).

\subsection{Quantitative Evaluation}

The concept of aesthetics is quite an abstract concept to be assessed without knowing an individual's preference. Hence, this article applies a compromising evaluation method to measure the image's colorfulness, since colorfulness plays a significant role in appealing to the human visual sense. Hence, one of the key properties of aesthetic enhancement is to improve its colorfulness. To produce an objective measure, an existing colorfulness evaluation method [42] is applied for the 13 previously introduced experiments. This quantitative evaluation is calculated based on Equation (11).

$$
Q=\sqrt{\sigma_{y b}^{2}+\sigma_{r g}^{2}}+0.3 \cdot \sqrt{\mu_{y b}^{2}+\mu_{r g}^{2}}
$$

where $\sigma_{y b}, \sigma_{r g}$ are the standard deviations of $y b$ and $r g$, and $\mu_{y b}, \mu_{r g}$ are mean value of $y b$ and $r g$, where $y b=\frac{R+G}{2}-B, r g=R-G$ for a color image in the $R G B$ color model.

For this metric, a larger colorfulness score means more colorfulness. For this article, the colorfulness measure results for both the original images and the recolored images are listed in Table 1. From the data in Table 1, the CT-AEA successfully improved the colorfulness for each original image with an average performance improvement ratio of $21.43 \%$. These results demonstrate the effectiveness of CT-AEA on improving colorfulness, meaning enhancing one aspect of aesthetics.

\subsection{User Study Evaluation}

Besides the previous quantitative evaluation, a subjective user study is also applied. In each test group, the volunteer was presented with an original image and a processed image. Since different people may express different opinions about photo aesthetic enhancement, this user study is conducted by asking two specific questions, including "Q1: Does the change between the original image and the processed image have an aesthetic effect on the photo?" And "Q2: Do you like the resulting aesthetic effect?" There are 13 test groups from the experimental results. The survey data was listed in Table 2 to present the number of Yes responses to each question out of the total 13 test groups for each volunteer. From the data, there are two observations: First, most of the volunteers think the modification by the proposed CT-AEA has an aesthetic effect. However, the ratio of volunteers liking this photo aesthetic effect is only $50 \%$. This can be explained by the different aesthetic preferences of the volunteers, which also shows the complexity and diversity of humans' aesthetic perception. Hence, the user study only demonstrates the effectiveness of this photo aesthetic effect editing in providing some artistic effects. 


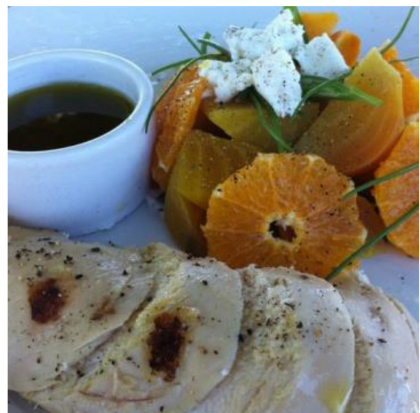

(a)

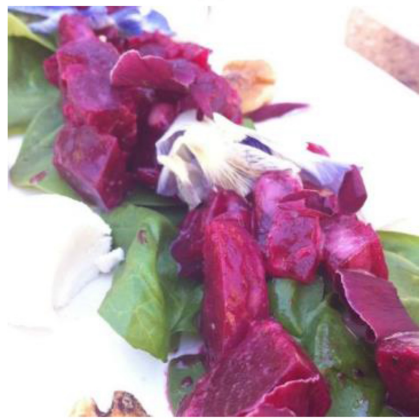

(c)

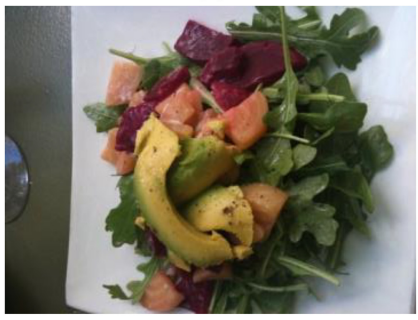

(e)

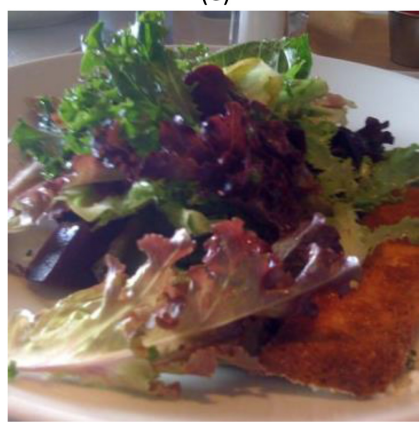

(g)

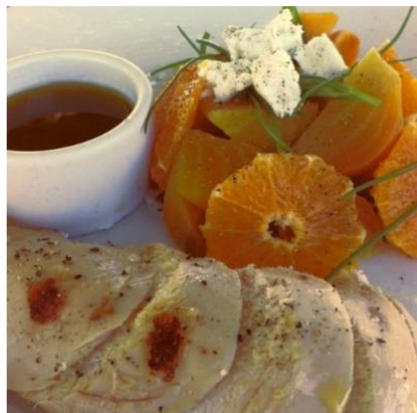

(b)

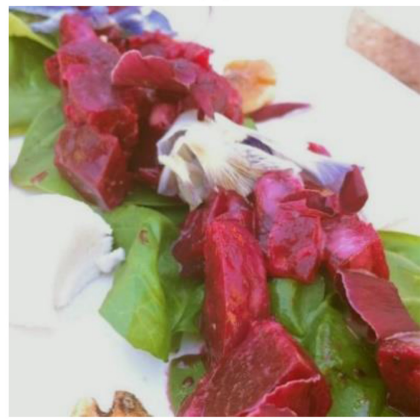

(d)

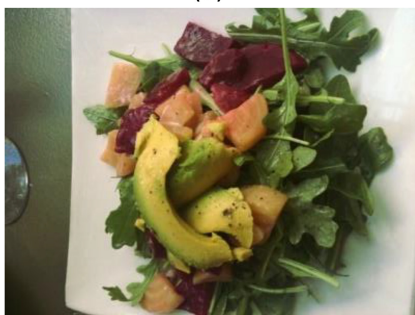

(f)

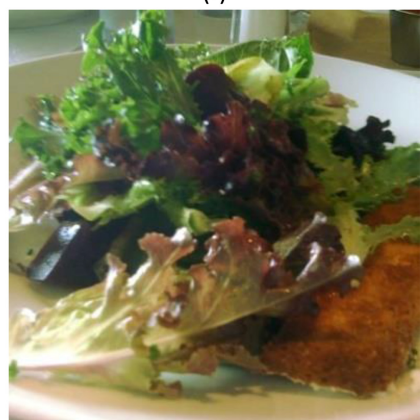

(h)

Fig. 5. Simulation result of food photography: (a), (c), (e), and (g) are original images [41]; (b) recolored result of (a) to present a yellower color of orange; (d) recolored result of (c) presenting the fresh green of vegetable and fresh red of beef; (f) recolored result of (e) showing the fresh green of both vegetable and avocado; (h) recolored result of $(\mathrm{g})$ showing the fresh green of salad vegetable, demonstrating the performance of CT-AEA in enriching the colorfulness. 
Table 1. Colorfulness Measure

\begin{tabular}{ccccc}
\hline $\begin{array}{c}\text { Test } \\
\text { Index }\end{array}$ & $\begin{array}{c}\text { Original } \\
\text { Image }\end{array}$ & $\begin{array}{c}\text { Original } \\
\text { Image }\end{array}$ & $\begin{array}{c}\text { Recolored } \\
\text { Image }\end{array}$ & $\begin{array}{c}\text { Improvement ratio } \\
\text { by CT-AEA (\%) }\end{array}$ \\
\hline 1 & Fig. 1(c) & 72.98 & 84.61 & 15.94 \\
2 & Fig. 3(a) & 32.98 & 33.26 & 0.85 \\
3 & Fig. 3(c) & 30.40 & 31.88 & 4.87 \\
4 & Fig. 3(e) & 28.58 & 36.11 & 26.35 \\
5 & Fig. 3(g) & 18.51 & 26.10 & 41.00 \\
6 & Fig. 4(a) & 48.02 & 59.04 & 22.95 \\
7 & Fig. 4(c) & 28.25 & 33.66 & 19.15 \\
8 & Fig. 4(e) & 16.63 & 27.66 & 66.32 \\
9 & Fig. 4(g) & 43.02 & 56.37 & 31.03 \\
10 & Fig. 5(a) & 51.71 & 59.06 & 14.20 \\
11 & Fig. 5(c) & 58.19 & 62.79 & 7.91 \\
12 & Fig. 5(e) & 38.76 & 43.96 & 13.41 \\
13 & Fig. 5(g) & 38.29 & 43.86 & 14.55 \\
\hline
\end{tabular}

Table 2. User Study (Q1: Does the change between original image and processed image have an aesthetic effect on the photo? Q2: Do you like the resulting aesthetic effect?)

\begin{tabular}{ccc}
\hline Volunteer & $\begin{array}{c}\text { Q1 } \\
\text { (Yes/test groups) }\end{array}$ & $\begin{array}{c}\text { Q2 } \\
\text { (Yes/test groups) }\end{array}$ \\
\hline 1 & $7 / 13$ & $2 / 13$ \\
2 & $6 / 13$ & $4 / 13$ \\
3 & $8 / 13$ & $7 / 13$ \\
4 & $9 / 13$ & $7 / 13$ \\
5 & $11 / 13$ & $9 / 13$ \\
6 & $12 / 13$ & $9 / 13$ \\
7 & $12 / 13$ & $6 / 13$ \\
8 & $7 / 13$ & $3 / 13$ \\
9 & $10 / 13$ & $5 / 13$ \\
10 & $13 / 13$ & $9 / 13$ \\
11 & $13 / 13$ & $10 / 13$ \\
12 & $10 / 13$ & $8 / 13$ \\
13 & $8 / 13$ & $8 / 13$ \\
14 & $8 / 13$ & $5 / 13$ \\
15 & $8 / 13$ & $6 / 13$ \\
16 & $6 / 13$ & $6 / 13$ \\
Average & $\mathbf{7 1 . 2 \%}$ & $\mathbf{5 0 \%}$ \\
\hline
\end{tabular}

\subsection{Fashion design Photography}

One of the key features in fashion design is diversity in color themes. To generate different recolored results with a different color theme, the original CT-AEA algorithm was extended. Instead of determining one single optimal color theme by Equation (4), the modification aims to recommend 

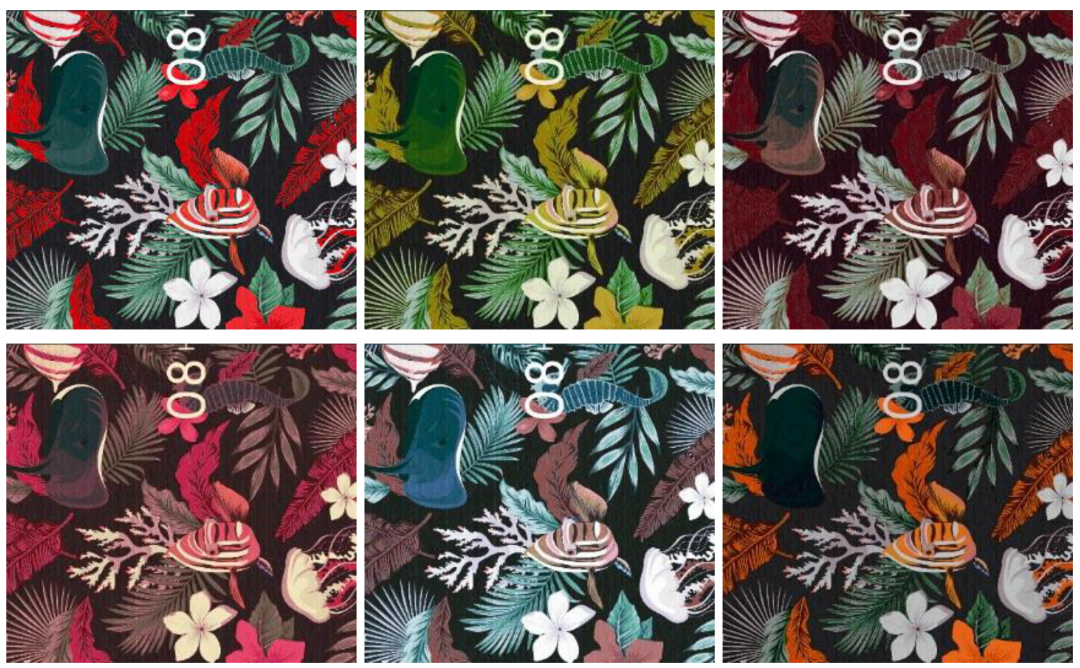

Fig. 6. Simulation result of fashion photography in graphic design. The top-left image is the original image, whereas the rest of the images are recolored by CT-AEA. ${ }^{5}$

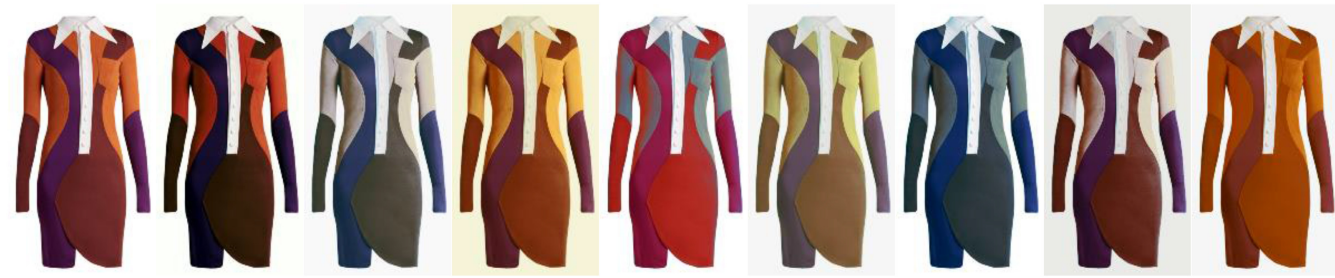

Fig. 7. Simulation result of fashion photography in clothes design. The leftmost image is the original image, whereas the rest of the images are recolored by CT-AEA. ${ }^{6}$

more good color themes as shown in Equation (11).

$$
\exists T_{N}^{*} \in \widehat{\mathrm{T}} \underset{T_{N}^{*}}{\arg \min } \mathcal{D}\left(T^{*}, T\right)-\mathcal{H}\left(T^{*}\right),
$$

where $T_{N}^{*}$ represents $N$ recommended theme matrixes. Each theme matrix contains five dominant colors, where each color is represented by three R, G, B component values, in the theme dataset $\hat{\mathrm{T}}$; Function $\operatorname{minN}$ aims at finding $N$ smallest value.

These recommended color themes not only generate different recolored images but also ensure that each recolored image has a certain aesthetic level and color harmony. As shown in Figures 6 and 7, these different recolored results provide more good design options. Designers or customers can select the results they like.

\subsection{Discussion}

As presented in the previous experimental results, CT-AEA meets the goals of supporting laymen users to simply and effectively enhance the aesthetic level of their photos. These experimental

\footnotetext{
${ }^{5}$ The original image is cropped from an image downloaded from https://d27yqot8savz5t.cloudfront.net/screengrabs/ images/000/009/985/medium.jpg.

${ }^{6}$ The original image is downloaded from https://seedesignmake.com/design-sew-givenchy-inspired-rugby-dress/
} 
results also show the diversity of applications for CT-AEA in many color-related art and design applications, including: art photography to elicit a strong emotional feeling from the human observer; fashion design photography to sell a lifestyle or present its aesthetic claim; commercial photography to sell a product by its appealing appearance; travel photography to attract tourists by showing the beauty of a natural scene; and food photography to entice the viewer's appetite. Furthermore, this algorithm tracks humans' aesthetic preference over time by analyzing color theme-related big data utilizing machine-learning technologies. However, this method only changes the color appearance and cannot change other aesthetic-related components, such as the geometric structure of the image contents and luminance distribution. It is our future plan to build an overall system for aesthetic enhancement by including all the aesthetic-related components.

\section{COMPARISON ANALYSIS}

To the best of our knowledge, there are no existing methods or systems that explore a photo's original inherent aesthetic feeling and accentuate it. The only similar color-editing method is color transfer-based approach for recoloring. Here, two color transfer-based methods, including Method [28] by Wang et al. and Method [30] by Phan et al., are compared to the CT-AEA. Their results are shown in Figures 8(c)-(d). Both these methods can generate the recolored images with different themes by manually selecting different combinations of source colors. Hence, there is a need for users to have the skills and experience to select the proper source colors for recoloring. If the source colors are improperly selected, then the generated images might present some unnatural or unpleasing feeling, e.g., colors are inharmonious when used in combination, rather than the desired aesthetic feeling. And most importantly, even though color transfer-based methods can generate recolored images with different aesthetic feelings, they do not explore the inner aesthetic ambiance of the original images and present it in a much more impactful way. Furthermore, the color inconsistency resulting from the color transfer operation with multiple source colors decreased the images' quality in Method [29], as shown in the red circle of the "Chase" image in Figure 8.

Compared to color transfer-based methods, the recolored image in Figure 8(b) visually verifies the CT-AEA's advantages in (1) requiring no manual process to select a proper source color for an aesthetically impactful purpose; (2) automatically exploring the inherent aesthetic feeling of the input photo; (3) successfully accentuating the image's inherent aesthetic feeling by adjusting its original color theme to achieve aesthetic enhancement; (4) ensuring the high quality of the output photos without the existence of color inconsistency.

Existing objective quality measures perform well at evaluating image distorted-related quality rather than image aesthetic-related quality $[43,44]$. Hence, to evaluate the users' feedback about this comparison test, a user study is conducted to let the users select the best aesthetic effect they like. The survey data is listed in Table 3. From this data, the CT-AEA and Method [28] with the color theme used in Figure 8(f) receives the highest scores; 8 and 6 respectively denote the first choice selections out of the total 16 volunteers. However, the data for Figure 8(c) and Figure 8(f), which shares the same Method [28] with a different color theme input, show it is extremely important for users to pick up the appropriate color theme. Hence, CT-AEA is quite convenient for users to apply the aesthetic enhancement, since the color theme is automatically optimized by the algorithm.

\section{CONCLUSIONS}

In this article, a new color theme-based approach was proposed, analyzed, and implemented by the introduced CT-AEA algorithm. This method differs from existing composite-based and 


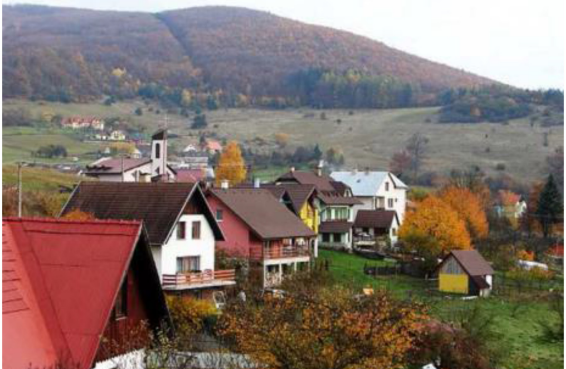

(a)

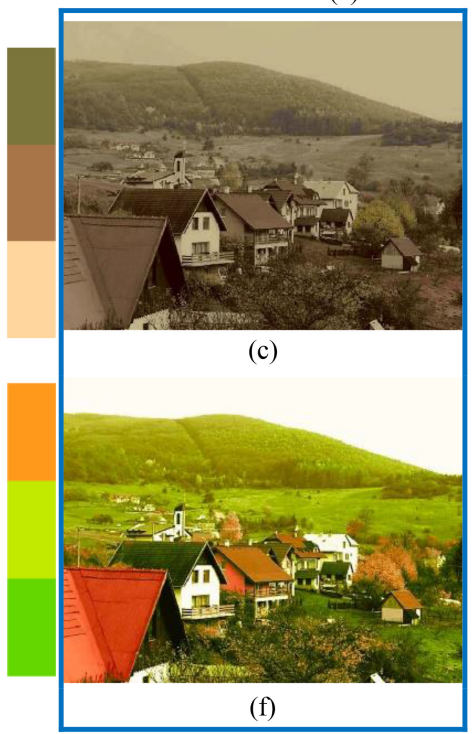

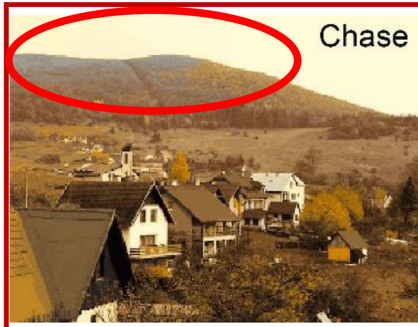

(d)

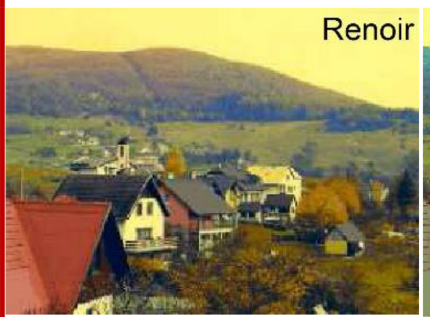

(g)

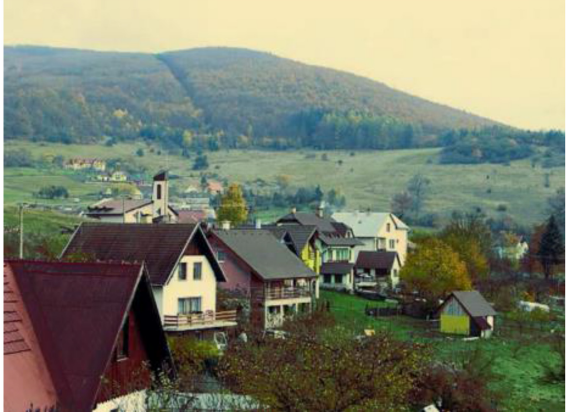

(b)

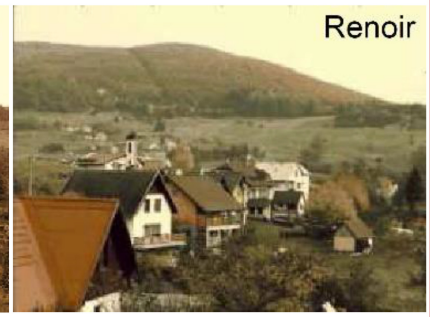

(e)

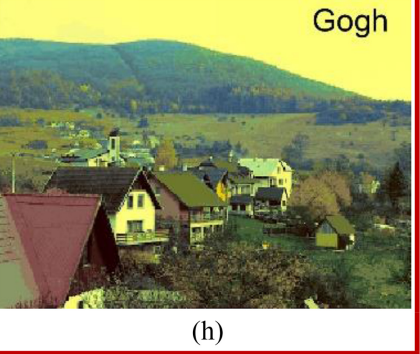

Fig. 8. Comparison analysis between two color transfer-based aesthetic enhancement methods (including Method [28] and Method [29]) and the proposed color theme-based CT-AEA method: (a) original image; (b) the result of the CT-AEA; (c) and (f) are the results of Method [28] with the users' source color setting as shown in the left-side key of each row; (d), (e), (g), and (h) are the results of Method [29] using three different artistic effects, including "Chase" effect, "Renoir" effect, and "Gogh," show that only the CT-AEA is exploring the inherent aesthetic feeling without the manual interaction, whereas the other two methods need to interface with the users. Note, it is extremely arduous and difficult for ordinary users to select the proper source colors to achieve some aesthetic design purpose without them possessing the background knowledge of color theory. This example also shows the limitation in the color consistency in image content details, which is highlighted by the red circle in the "Chase" image.

color/luminance-related approaches by leveraging the color theme's effect on human aesthetic perception. Unlike existing color/luminance-related approaches that recolor the image to present a different aesthetic feeling, this approach aims at exploring and accentuating the image's original aesthetic feeling. This new CT-AEA algorithm learned from humans' aesthetic experience from a large online dataset and applied that learned knowledge to aesthetic enhancement by recommending an optimal color theme for recoloring. By updating the color theme of original images with the recommended optimal color theme, the CT-AEA approach maximizes aesthetic improvement 
Table 3. User Study Among Comparison Results in Figure 8 to Select the Best Aesthetic Effect the 16 Volunteers Like

\begin{tabular}{lcc}
\hline Method & Volunteer & $\begin{array}{c}\text { \# of First Choice } \\
\text { Selection / \# of volunteers }\end{array}$ \\
\hline Method [28] & Fig. 8(c) & $1 / 16$ \\
& Fig. 8(f) & $6 / 16$ \\
Method [29] & Fig. 8(d) & $1 / 16$ \\
& Fig. 8(e) & - \\
& Fig. 8(g) & - \\
CT-AEA & Fig. 8(h) & - \\
\hline
\end{tabular}

with a minimized color-modification cost. Experimental results demonstrated its performance, and a quantitative measure was used to validate the visual assessment claims of improvement.

\section{REFERENCES}

[1] Y. Zhang, L. Zhang, and R. Zimmermann. 2015. Aesthetics-guided summarization from multiple user generated videos. ACM Trans. Multimedia Comput. Commun. Appl. 11 (2015), 1-23.

[2] C.-H. Yeh, B. A. Barsky, and M. Ouhyoung. 2014. Personalized photograph ranking and selection system considering positive and negative user feedback. ACM Trans. Multimedia Comput. Commun. Appl. 10 (2014), 1-20.

[3] R. Schifanella, M. Redi, and L. Aiello. 2015. An image is worth more than a thousand favorites: Surfacing the hidden beauty of Flickr pictures. In Proceedings of the International AAAI Conference on Web and Social Media.

[4] J. Chen, G. Bai, S. Liang, and Z. Li. 2016. Automatic image cropping: A computational complexity study. In Proceedings of the IEEE Conference on Computer Vision and Pattern Recognition (CVPR'16). 507-515.

[5] R. Datta, D. Joshi, J. Li, and J. Z. Wang. 2006. Studying Aesthetics in Photographic Images Using a Computational Approach. Springer Berlin, 288-301.

[6] R. Hong, L. Zhang, and D. Tao. 2016. Unified photo enhancement by discovering aesthetic communities from Flickr. IEEE Trans. Image Proc. 25 (2016), 1124-1135.

[7] Y. Deng, C. C. Loy, and X. Tang. 2017. Aesthetic-Driven image enhancement by adversarial learning. Retrieved from: arXiv preprint arXiv:1707.05251.

[8] S. Bhattacharya, R. Sukthankar, and M. Shah. 2011. A holistic approach to aesthetic enhancement of photographs. ACM Trans. Multimedia Comput. Commun. Appl. 7S (2011), 1-21.

[9] M. Nishiyama, T. Okabe, I. Sato, and Y. Sato. 2011. Aesthetic quality classification of photographs based on color harmony. In Proceedings of the Conference on Computer Vision and Pattern Recognition (CVPR'11). 33-40.

[10] L. Wei, W. Xiaogang, and X. Tang. 2011. Content-based photo quality assessment. In Proceedings of the International Conference on Computer Vision. 2206-2213.

[11] J. Yan, S. Lin, S. B. Kang, and X. Tang. 2013. Learning the change for automatic image cropping. In Proceedings of the IEEE Conference on Computer Vision and Pattern Recognition. 971-978.

[12] W. Wang, J. Shen, and L. Shao. 2015. Consistent video saliency using local gradient flow optimization and global refinement. IEEE Trans. Image Proc. 24 (2015), 4185-4196.

[13] W. Wang, J. Shen, L. Shao, and F. Porikli. 2016. Correspondence driven saliency transfer. IEEE Trans. Image Proc. 25 (2016), 5025-5034.

[14] Y. Gao, J. Guo, T. Ren, and J. Bei. 2016. Personal photo enhancement via saliency driven color transfer. In Proceedings of the International Conference on Internet Multimedia Computing and Service.

[15] M. B. Islam, W. Lai-Kuan, and W. Chee-Onn. 2017. A survey of aesthetics-driven image recomposition. Multimedia Tools Appl. 76 (2017), 9517-9542.

[16] M. B. Islam, W. Lai-Kuan, W. Chee-Onn, and K. L. Low. 2015. Stereoscopic image warping for enhancing composition aesthetics. In Proceedings of the 3rd IAPR Asian Conference on Pattern Recognition (ACPR'15). 645-649.

[17] K. Jeong and H.-J. Cho. 2016. Photo quality enhancement by relocating subjects. Cluster Comput. 19 (2016), 939-948.

[18] L. Liu, R. Chen, L. Wolf, and D. Cohen-Or. 2010. Optimizing photo composition. Comput. Graph. Forum. 29, 2 (2010), 469-478.

[19] Y. W. Guo, M. Liu, T. T. Gu, and W. P. Wang. 2012. Improving photo composition elegantly: Considering image similarity during composition optimization. Comput. Graph. Forum 31, 7 (2012) 2193-2202. 
[20] L. Liu, Y. Jin, and Q. Wu. 2010. Realtime aesthetic image retargeting. In Proceedings of the 6th International Conference on Computational Aesthetics in Graphics, Visualization and Imaging.

[21] F. L. Zhang, M. Wang, and S. M. Hu. 2013. Aesthetic image enhancement by dependence-aware object recomposition. IEEE Trans. Multimedia 15 (2013), 1480-1490.

[22] S. H. Lim and D. A. Silverstein. 2016. Image tone adjustment using local tone curve computation, S. H. Lim and D. A. Silverstein (Eds.). U.S. Patent 9369684. Retrieved from https://patents.google.com/patent/US9369684B2/en?q= Image \&q=tone \&q=adjustment $\& \mathrm{q}=$ local $\& \mathrm{q}=$ tone $\& \mathrm{q}=$ curve $\& \mathrm{q}=$ computation $\&$ oq=Image+tone+adjustment+using+ local+tone+curve+computation.

[23] J. Choi, S. Koh, J. Kwack, Y. Kwon, and H. Shim. 2016. Data-driven approach to aesthetic enhancement. Electron. Imag. 14 (2016), 1-5.

[24] B. Wang, Y. Yu, and Y.-Q. Xu. 2011. Example-based image color and tone style enhancement. ACM Trans. Graph. 30 (2011), 1-12.

[25] L. Bao, K. Panetta, and S. Agaian. 2016. A new color transfer quality measure. In Proceedings of SPIE 9869, Mobile Multimedia/Image Processing, Security, and Applications 2016, 986904.

[26] K. Panetta, L. Bao, and S. Agaian. 2016. Novel multi-color transfer algorithms and quality measure. IEEE Trans. Consum. Electron. 62 (2016), 292-300.

[27] L. Bao, K. Panetta, and S. Agaian. 2017. Fast color transfer for camouflage applications. In Proceedings of the IEEE International Symposium on Technologies for Homeland Security (HST'17). 1-5.

[28] B. Wang, Y. Yu, T.-T. Wong, C. Chen, and Y.-Q. Xu. 2010. Data-driven image color theme enhancement. ACM Trans. Graph. 29 (2010), 1-10.

[29] H. Q. Phan, H. Fu, and A. B. Chan. 2018. Color orchestra: Ordering color palettes for interpolation and prediction. IEEE Trans. Vis. Comput. Graph. 24 (2018), 1942-1955.

[30] C. C. Gramazio, D. H. Laidlaw, and K. B. Schloss. 2017. Colorgorical: Creating discriminable and preferable color palettes for information visualization. IEEE Trans. Vis. Comput. Graph. 23 (2017), 521-530.

[31] K. Naz and H. Epps. 2004. Relationship between color and emotion: A study of college students. Coll. Stud. F. 38 (2004), 396.

[32] P. T.-F. Chong, C. Minchew, and K. Ewing. 2015. Color selection system based on desired color emotion and color harmony, P. T.-F. Chong, C. Minchew, and K. Ewing. U.S. Patent No. 9134179. Retrieved from https://patents.google. com/patent/US9134179B2/en.

[33] S. Liu and H. Luo. 2016. Hierarchical emotional color theme extraction. Color Res. Appl. 41 (2016), 513-522.

[34] P. O’Donovan, A. Agarwala, and A. Hertzmann. 2014. Collaborative filtering of color aesthetics. In Proceedings of the Workshop on Computational Aesthetics.

[35] P. O'Donovan, A. Agarwala, and A. Hertzmann. 2011. Color compatibility from large datasets. In Proceedings of the ACM Transactions on Graphics 30, 4 (2011), 63.

[36] J. Nayak, B. Naik, and H. Behera. 2015. Fuzzy C-means (FCM) clustering algorithm: A decade review from 2000 to 2014. In Computational Intelligence in Data Mining, Vol. 2. Springer, 133-149.

[37] C. Liu, W. Huang, F. Sun, M. Luo, and C. Tan. 2019. LDS-FCM: A linear dynamical system based fuzzy c-means method for tactile recognition. IEEE Trans. Fuzzy Syst. 27 (2019), 72-83.

[38] J. F. Mota, L. Weizman, N. Deligiannis, Y. C. Eldar, and M. R. Rodrigues. 2017. Reweighted l1-norm minimization with guarantees: An incremental measurement approach to sparse reconstruction. In Proceedings of the 6th Signal Processing with Adaptive Sparse Structured Representations Workshop (SPARS'17).

[39] A. Olmos and F. A. A. Kingdom. 2004. A biologically inspired algorithm for the recovery of shading and reflectance images. Perception 33 (2004), 1463-1473.

[40] L. Bossard, M. Guillaumin, and L. Van Gool. 2014. Food-101-Mining discriminative components with random forests. In Proceedings of the European Conference on Computer Vision. 446-461.

[41] K. Lant. 2017. Colors in marketing and advertising. 99designs. Retrieved from https://99designs.com/blog/tips/ colors-marketing-advertising/.

[42] D. Hasler and S. Süsstrunk. 2003. Measuring colourfulness in natural images. In Proceedings of the Conference on Human Vision and Electronic Imaging VIII.

[43] L. Bao, K. Panetta, and S. Agaian. 2015. A no reference image quality measure using a distance doubling variance. In Proceedings of the IEEE International Conference on Technologies for Practical Robot Applications (TePRA'15). 1-6.

[44] K. Panetta, L. Bao, and S. Agaian. 2016. A human visual no-reference image quality measure. IEEE Instrument. Measur. Magazine 19 (2016), 34-38.

Received August 2018; revised February 2019; accepted April 2019 